\title{
Nebulization Solution Dosage Form
}

National Cancer Institute

\section{Source}

National Cancer Institute. Nebulization Solution Dosage Form. NCI Thesaurus. Code C149694.

Liquid preparation consisting of a solution intended for conversion by nebulizers into an aerosol. 